\title{
Identifying Significant Changes in Cerebrovascular Reactivity to Carbon Dioxide
}

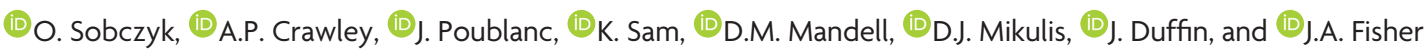

\section{ABSTRACT}

BACKGROUND AND PURPOSE: Changes in cerebrovascular reactivity can be used to assess disease progression and response to therapy but require discrimination of pathology from normal test-to-test variability. Such variability is due to variations in methodology, technology, and physiology with time. With uniform test conditions, our aim was to determine the test-to-test variability of cerebrovascular reactivity in healthy subjects and in patients with known cerebrovascular disease.

MATERIALS AND METHODS: Cerebrovascular reactivity was the ratio of the blood oxygen level-dependent MR imaging response divided by the change in carbon dioxide stimulus. Two standardized cerebrovascular reactivity tests were conducted at $3 \mathrm{~T}$ in 15 healthy men (36.7 \pm 16.1 years of age) within a 4-month period and were coregistered into standard space to yield voxelwise mean cerebrovascular reactivity interval difference measures, composing a reference interval difference atlas. Cerebrovascular reactivity interval difference maps were prepared for 11 male patients. For each patient, the test-retest difference of each voxel was scored statistically as z-values of the corresponding voxel mean difference in the reference atlas and then color-coded and superimposed on the anatomic images to create cerebrovascular reactivity interval difference z-maps.

RESULTS: There were no significant test-to-test differences in cerebrovascular reactivity in either gray or white matter (mean gray matter, $P=.431$; mean white matter, $P=.857$; paired $t$ test) in the healthy cohort. The patient cerebrovascular reactivity interval difference $z$-maps indicated regions where cerebrovascular reactivity increased or decreased and the probability that the changes were significant.

CONCLUSIONS: Accounting for normal test-to-test differences in cerebrovascular reactivity enables the assessment of significant changes in disease status (stability, progression, or regression) in patients with time.

ABBREVIATIONS: $\mathrm{BOLD}=$ blood oxygen level-dependent; $\mathrm{CO}_{2}=$ carbon dioxide; $\mathrm{CVR}=$ cerebrovascular reactivity; $\mathrm{EC}-\mathrm{IC}=$ extracranial to intracranial; $\mathrm{ID}=$ interval difference; $\mathrm{PaCO}_{2}=$ arterial partial pressure of carbon dioxide; $\mathrm{PetCO}_{2}=$ end-tidal partial pressure of carbon dioxide

$\mathbf{K}_{\mathrm{n}}^{\mathrm{n}}$ nowledge of the repeatability of any test is crucial for assessing not only the progress of a disease with time but also the effects of any therapeutic intervention. Cerebrovascular reactivity (CVR) is a test indicating the magnitude of CBF responses to a

Received July 22, 2015; accepted after revision October 23.

From the Institute of Medical Science (O.S., D.J.M., J.A.F.) and Department of Physiology (K.S., J.D., J.A.F.), University of Toronto, Toronto, Canada; and Joint Department of Medical Imaging and the Functional Neuroimaging Laboratory (A.P.C., J.P. K.S., D.M.M., D.J.M.) and Department of Anaesthesia and Pain Management (J.D., J.A.F.), University Health Network, Toronto, Canada.

J.A.F. is Chief Scientist and J.D. is Senior Scientist at Thornhill Research, a spin-off company from the University Health Network that developed the RespirAct. RespirAct is currently a noncommercial research tool assembled and made available by Thornhill Research to research institutions to enable CVR studies.

Please address correspondence to Olivia Sobczyk, MSc, Institute of Medical Science, Medical Sciences Building, One King's College Circle, University of Toronto, Toronto, ON, Canada, M5S 1A8; e-mail: o.pucci@mail.utoronto.ca

Indicates article with supplemental on-line table.

Indicates article with supplemental on-line photo.

http://dx.doi.org/10.3174/ajnr.A4679 vasoactive stimulus. Reductions in CVR have been shown to indicate an enhanced risk of stroke ${ }^{1-3}$ and reduced cognitive ability. ${ }^{4}$ There are currently a number of techniques for mapping CVR. We have found blood oxygen level-dependent (BOLD) MR imaging signal intensity during arterial partial pressure of carbon dioxide $\left(\mathrm{PaCO}_{2}\right)$ manipulation a reliable measure of CVR. Our CVR test is, in effect, a cerebrovascular "stress test," in which occult regional limitations of hemodynamic reserve can be mapped using MR imaging of a stimulated global increase in $\mathrm{CBF}^{5-7}$

\section{Clinical Aspects of CVR Maps}

Clinically significant changes in CVR maps must be distinguishable from the normal variation in CVR due to equipment variability, artifacts such as patient movement, and normal day-today physiologic variability. If we consider a longitudinal study of a group of patients undergoing an intervention in which we wish to test the effectiveness of the intervention, performing a paired $t$ test 
would account for this background variability. In the case of a single patient whose improvement in CVR after an intervention is to be evaluated, we used a group of healthy control subjects to provide an equivalent statistical yardstick to test the significance of the measured change in CVR relative to the expected reproducibility of the test. To that end, we have standardized the $\mathrm{PaCO}_{2}$ stimulus, a critical component for developing test-to-test reliability, MR imaging sequence parameters, and data analysis. ${ }^{8-10}$ Once this step has been performed, quantization of the unavoidable background variability in CVR "noise" can be ascertained. We hypothesize that the variations in CVR outside the range of these normal test-to-test changes will be attributable to pathophysiologic changes.

\section{Study Aim}

Therefore, our aim in this study was to develop and test a quantitative method for detecting significant within-subject changes in CVR with time. We created a voxelwise CVR test-to-test interval difference (ID) atlas for healthy subjects (CVR ID atlas). We used the SD of the CVR ID atlas to score the probability that the voxelwise differences between 2 CVR studies for a patient would exceed the normal variation (ie, $z$ scores). We report on the use of this method to monitor the changes with time in CVR in 11 patients with intracranial steno-occlusive disease, most of whom had undergone revascularization procedures.

\section{MATERIALS AND METHODS \\ Subjects and Ethics Approval}

All studies conformed to the standards set by the latest revision of the Declaration of Helsinki and were approved by the Research Ethics Board of the University Health Network and Health Canada. All subjects gave written and informed consent. We recruited 15 healthy male subjects (mean age, $36.7 \pm 16.1$ years), with no history of neurologic or cardiovascular disease, who were nonsmokers and were taking no medication, to generate the CVR ID atlas described below. They were asked not to engage in heavy exercise or drink caffeinated drinks on the day of the test. For the patient examples, we searched our database of the research ethics board-approved CVR studies in male subjects (to minimize hormonal effects on CVR) with chronic cerebrovascular disease $(n=$ 11) who had undergone $\geq 2$ CVR examinations.

\section{Experimental Protocol}

Hypercapnic Stimulus. Subjects were fitted with a facemask and connected to a sequential gas-delivery breathing circuit. ${ }^{11}$ The patterns of end-tidal partial pressure of carbon dioxide $\left(\mathrm{PetCO}_{2}\right)$ and end-tidal partial pressure of oxygen were programmed into the automated gas blender (RespirAct; Thornhill Research, Toronto, Ontario, Canada) running the prospective gas-targeting algorithm of Slessarev et al. ${ }^{12}$ A standardized step carbon dioxide $\left(\mathrm{CO}_{2}\right)$ stimulus sequence was implemented, consisting of the following: a baseline $\mathrm{PetCO}_{2}$ of $40 \mathrm{~mm} \mathrm{Hg}$ for 60 seconds, step to a hypercapnia of $50 \mathrm{~mm} \mathrm{Hg}$ for 45 seconds, baseline for 90 seconds, hypercapnia for 120 seconds, and return to baseline for 60 seconds, all during isoxic normoxia. The implementation of prospective end-tidal gas control is described in greater detail by Slessarev et al. ${ }^{12}$
Table 1: Duration between CVR T1 scan and CVR T2 scan for each of the 15 healthy subjects in the ID CVR atlas

\begin{tabular}{cc}
\hline Subject No. & Time between T1 and T2 \\
\hline 1 & 12 Weeks \\
2 & 4 Weeks \\
3 & 8 Weeks \\
4 & 2 Weeks \\
5 & 4 Weeks \\
6 & 8 Weeks \\
7 & 16 Weeks \\
8 & 2 Weeks \\
9 & 4 Weeks \\
10 & 4 Weeks \\
11 & 16 Weeks \\
12 & 2 Weeks \\
13 & 4 Weeks \\
14 & 12 Weeks \\
15 & 2 Weeks \\
\hline
\end{tabular}

Note:-T1 indicates time point 1; T2, time point 2.

MR Imaging Protocol and CVR Map Generation. MR imaging was performed with a 3T scanner (Signa; GE Healthcare, Milwaukee, Wisconsin) with an 8-channel phased array head coil and consisted of BOLD acquisitions using a gradient-echo pulse sequence with an echo-planar readout (TR/TE, 2000/30 ms; FOV, $24 \times 24$ $\mathrm{cm}$; matrix size, $64 \times 64$; number of temporal frames, 254; 39 sections; section thickness, $5 \mathrm{~mm}$; no intersection gap; and flip angle, $85^{\circ}$ ). The acquired MR imaging and $\mathrm{PetCO}_{2}$ data were analyzed using Analysis of Functional NeuroImages software (AFNI; http://afni.nimh.nih.gov/afni). ${ }^{13} \mathrm{PetCO}_{2}$ data were timeshifted to the point of maximum correlation with the voxel showing the greatest positive BOLD signal change and were resampled at the TR. A linear, least-squares fit of the BOLD signal data series to the $\mathrm{PetCO}_{2}$ data series was then performed on a voxel-by-voxel basis. BOLD images were then volume-registered and section-time corrected and coregistered to an axial 3D T1-weighted inversion recovery fast-spoiled gradient-recalled volume (TI/TR/TE, 450/8/3 ms; matrix size, $256 \times 256$; FOV, $22 \times 22 \mathrm{~cm}$; section thickness, $1 \mathrm{~mm}$; and flip angle, $15^{\circ}$ ), which was acquired in the same imaging session. ${ }^{14}$ The slope of the linear relation between the BOLD signal and the $\mathrm{PetCO}_{2}$ was color-coded to a spectrum of colors corresponding to the direction (positive or negative) and the magnitude of the correlation and overlaid on the corresponding structural scans to form CVR maps. All voxels with correlation coefficients between -0.125 and +0.125 were thresholded from the maps. All subjects and patients had $<3 \mathrm{~mm}$ head movement in any direction during the scanning.

\section{Analyzing the CVR Maps}

Repeatability and Construction of the ID CVR Atlas. The 15 healthy subjects underwent 2 CVR measurements within a 4-month interval to mimic the times usually implemented for our patient CVR measurements pre- and postsurgical intervention (see Table 1 for between-study time intervals for the healthy subjects). Regional measures of CVR were obtained by segmenting the anatomic images into gray and white matter regions (SPM8 software; http://www.fil.ion.ucl.ac.uk/spm/software/spm8).

Construction of the CVR ID atlas proceeded by first coregistering all datasets into Montreal Neurological Institute standard 

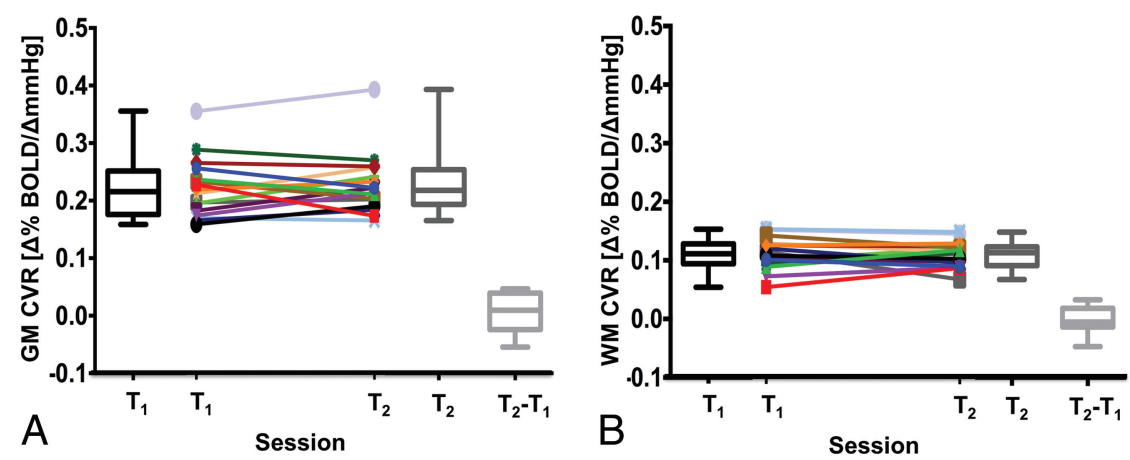

FIG 1. Comparison of CVR test 1 and CVR test 2 for gray matter $(A)$ and white matter $(B)$ for each of the 15 healthy subjects in the ID CVR atlas. There were no significant differences between tests (mean gray matter, $P=.431$; mean white matter, $P=.857$ ). The horizontal line in the box represents the median, the box represents the interquartile range ( $25 \%$ to $75 \%$ ), and the whiskers represent the minimum and maximum values. $\mathrm{Tl}$ indicates time point session 1; T2, time point session 2.

\begin{tabular}{|c|c|c|}
\hline & Gray Matter & White Matter \\
\hline Mean CVR ( $\Delta \%$ BOLD $/ \Delta \mathrm{mm} \mathrm{Hg})$ & $0.228(0.01)$ & $0.117(0.001)$ \\
\hline Mean CV (\%) & $7.3(1.33)$ & $10.3(2.19)$ \\
\hline
\end{tabular}

space (as defined by a T1-weighted Montreal Neurological Institute 152 standard template $)^{15,16}$ and then by calculating a voxelwise difference in CVR values between the 2 time points in each of the 15 subjects. From these difference maps, a spatial smoothing of full width at half maximum at $5 \mathrm{~mm}$ was applied to each voxel. The difference, mean, and SD over all subjects for each voxel (AFNI software) ${ }^{13}$ were calculated to produce the test-to-test CVR ID atlas. Comparisons with time for systematic differences in the CVR scans were evaluated by using paired Student $t$ tests (significance, $P<.05$ ). Coefficients of variation for gray and white matter were evaluated as estimates of repeatability (SigmaPlot 12.5; Systat Software, San Jose, California).

CVR ID Z-Maps. After spatial normalization, test-to-test CVR differences were calculated for 10 male patients who underwent repeated CVR studies within a year (On-line Figure) and 1 male patient who underwent 5 CVR scans during a 3-year period, to produce their CVR ID maps. Because CVR changes little with age, we did not expect systematic differences in CVR in healthy subjects during the 4-month range of repeat studies, and indeed there were none $(P>.05$, paired Student $t$ test). The differences that we saw were due to small errors in coregistration, partial voluming effects, and day-to-day physiologic changes in the subjects and in the hardware. Because they do not represent real systematic differences, the mean ID values would trend to zero with larger subject cohorts. The assumption of zero as the sample mean difference produces an unbiased, estimate of the SD. The $z$ scores for these patients were calculated voxel-by-voxel as the difference between the patient CVR ID values and those of the reference cohort (ie, zero) divided by the CVR ID atlas SD. The resulting CVR ID $z$ scores indicate the statistical probability that the magnitude of the CVR interval differences was outside the normal range. These calculated CVR ID $z$ scores were then color-coded (On-line Figure and On-line Table ${ }^{10}$ ) and superimposed on the anatomic images to produce CVR ID z-maps showing the distribution and statistical significance of changes in the patient CVRs with time.

\section{RESULTS \\ Normal Repeatability}

Figure 1 presents the distribution of the mean CVR values for both gray and white matter for each CVR test in the 15 control subjects. The overall mean CVR and coefficient of variation reproducibility measures for gray and white matter are presented in Table 2. On average, CVR measures were repeatable in both gray $($ coefficient of variation $=7.3 \%$ ) and white matter (coefficient of variation $=10.3 \%)$, consistent with repeatability measurements found in a previous study using the same CVR measurement method. ${ }^{17}$ The paired Student $t$ test found no significant systematic differences between the CVR scans.

\section{ID Atlas Characteristics}

Figure 2 shows the spatial distribution of the CVR ID atlas mean and SD test-to-test differences. The mean differences (Fig $2 A$ ) are close to zero, with the highest differences located in the superior sagittal sinus. Mean CVR differences $( \pm$ standard error of the mean) in the ID atlas for gray matter were found to be $0.0057 \pm$ 0.013 and $0.0047 \pm 0.010$ for white matter. Areas with the highest test-to-test differences in the CVR ID atlas (Fig 2B) were found in the cortex and regions with large veins.

\section{ID Z-Map Examples}

Figure 3 shows an application of the CVR ID atlas to assess changes in CVR with time in a healthy subject (not included in the CVR ID atlas).

Figure 4 shows the application of the CVR ID atlas to assess changes in CVR with time in a patient drawn from our data base (more patient examples are shown in the On-line Figure and On-line Table) with right extracranial to intracranial (ECIC) bypass surgery. MR angiography shows complete right ICA occlusion and $90 \%$ left ICA stenosis preoperatively. Postoperatively, the patient continued to experience episodes of right hemisphere transient ischemic attacks. Their CVR map determined 1 month before surgery (Fig $4 A$ ) showed decreased CVR in the right hemisphere. CVR measured 4 months postoperatively (Fig 4B) shows improvement. The CVR ID z-map shows the distribution of the interval changes and indicates the probability that these changes are due to the normal test-to-test variability. The second follow-up CVR (Fig 4C) performed 3 months later shows regression of the CVR. Additional follow-up CVR studies (Fig $4 D,-E$ ) show that further changes in CVR were small and were mostly in the range of normal test-to-test variability. Figure $4 F$ compares study $A$ with study $E$, or the net change in CVR for the duration. It indicates that despite the regression of CVR in many areas following the operation, there are residual areas of increased CVR compared with the preoperative study. 

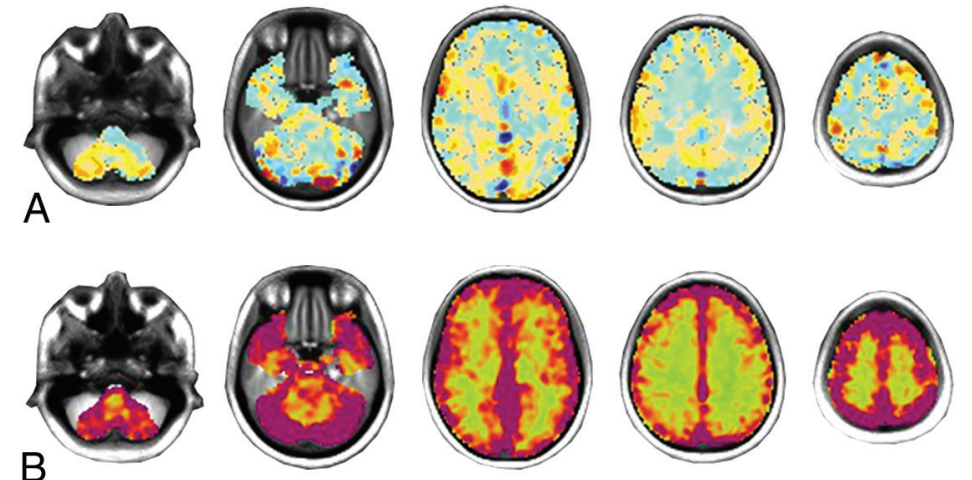

FIG 2. Axial sections displaying the spatial distribution of the healthy cohort. $A$, The mean difference in CVR between the 2 CVR tests colored according to the scale shown on the right in percentage change of BOLD per millimeter-of-mercury change in $\mathrm{PetCO}_{2} . \mathrm{B}$, The SD of CVR differences. The color scale is on the right.
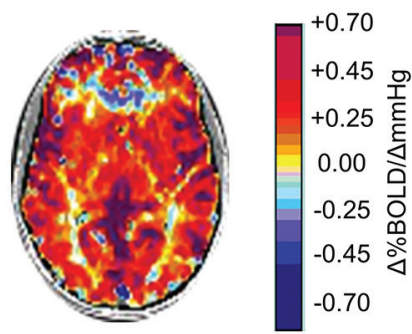

CVR map

Day 1

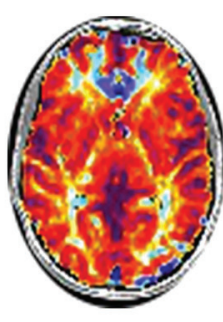

CVR map

Day 2
CVR ID z-map

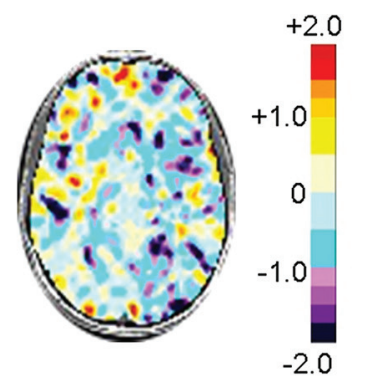

FIG 3. An axial section from the midbrain of a healthy subject's CVR maps measured 2 months apart. These CVR measurements show the spatial distribution of CVR values colored according to the scale shown (percentage change of BOLD per millimeter-of-mercury change in PetCO $\mathrm{O}_{2}$ ). The corresponding CVR ID z-map is shown on the right. The CVR ID z-map provides a perspective of the statistically normal differences in CVR with time.

\section{DISCUSSION}

\section{Identifying Significant Changes with Time}

In this article, we introduce a statistical analytic method for determining the voxelwise probability of a change in CVR between 2 scans: the CVR ID z-map. We minimized the test-to-test variability by standardizing what has previously been a major source of variability: the vasoactive stimulus. ${ }^{18}$ With most methods, the partial pressure of $\mathrm{CO}_{2}$ of the hypercapnic stimulus varies widely. ${ }^{19,20}$ In this study, the stimulus was a precisely ( $\pm 2 \mathrm{~mm}$ $\mathrm{Hg}$ ) repeatable change in $\mathrm{PaCO}_{2}$. In a previous study, a patient's CVR scan was scored relative to a reference atlas to identify abnormal regions. ${ }^{8}$ That was a cross-sectional atlas designed to score the probability of CVR values differing from normal in a single patient; in contrast, the present CVR ID atlas provides the significance of CVR changes between $\geq 2$ tests in a single patient with time. This capability of identifying changes between CVR tests is necessary for longitudinal studies, such as following the progress of disease and determining the effects of interventions.

\section{Minimizing Variability in CVR Tests due to Variation in the Stimulus}

Mapping cerebrovascular reactivity requires a vasoactive stimulus that applies to the entire brain. Infusing pharmacologic vasoactive agents such as acetazolamide results in a variability of blood levels with time and between subjects — even with standardized doses; other institution. ${ }^{27}$
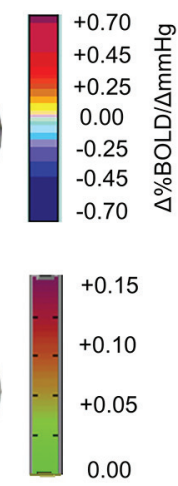

there is even a variation of the vasodilatory response to a given blood level (see Fierstra et $\mathrm{al}^{19}$ for a discussion). By contrast, using hypercapnia as the vasoactive stimulus ${ }^{21}$ can result in a more reliable response. The drawback of using hypercapnia as the vasoactive stimulus is the difficulty in repeatedly attaining a target $\mathrm{PaCO}_{2}$. Infusing $\mathrm{CO}_{2}$ into a face mask, ${ }^{22}$ inhaling a fixed concentration of $\mathrm{CO}_{2},{ }^{23}$ or simply breath-holding ${ }^{24}$ do not produce a reproducible stimulus and cannot even provide a reliable measure of the change in the $\mathrm{PaCO}_{2} \cdot{ }^{18,25}$ Therefore, we used a computer-controlled gas blender in this study to prospectively target a $\mathrm{PetCO}_{2}$ that was equilibrated with $\mathrm{PaCO}_{2} \cdot{ }^{26}$

This methodology enabled repeat administration of a standardized stimulus (from baseline $\mathrm{PetCO}_{2}$ at $40.2 \pm 1.1$ $\mathrm{mm} \mathrm{Hg}$ to $49.9 \pm 1.5 \mathrm{~mm} \mathrm{Hg}$ ) and thereby minimized the stimulus variability in the atlas and individual patient CVR tests. Our test is focused on showing that the repeatability of a CVR test provides the potential for clinical application, regardless of the type of stimulus used. The greater the variability in the stimulus, the larger the SD is for each voxel in the ID atlas and the lower the sensitivity for detecting changes in CVR. The versatility in this approach is that each institution, by standardizing its own acquisition sequences and vasoactive stimulus, can obtain its own custom reference atlas, and the $z$ scores of the institution would be comparable with those of any

\section{Accounting for Variability in CVR Tests due to Signal Variation}

Despite the precise designation of the MR imaging sequences and data analysis, there are nevertheless technical causes for variation in the CVR measurements. During acquisition, the BOLD signal can be affected by drift that has no consistent pattern or direction with time. In addition, there is day-to-day physiologic variability in vascular reactivity. The CVR ID atlas incorporates these variabilities so that CVR ID z-maps provide a confidence interval for identifying changes other than those attributable to these factors. The choice of thresholds for test-to-test difference determines the balance between sensitivity and specificity.

\section{Change in CVR with Time in Patients with Cerebrovascular Disease}

In the patients we studied (On-line Figure and On-line Table), the symptoms were mild and transient. In contrast, the obstructions to the vessels as seen on angiography were severe. The CVR mea- 


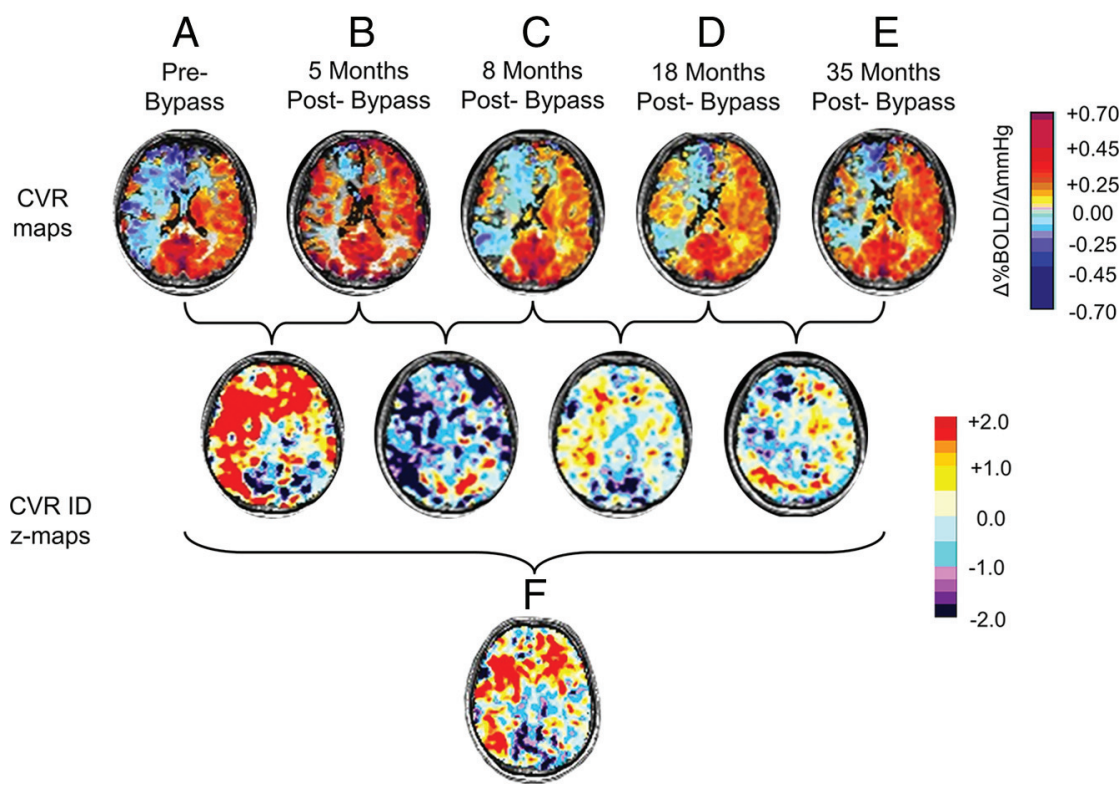

FIG 4. Longitudinal changes in CVR for a 56-year-old man with bilateral ICA stenosis who underwent right EC-IC bypass. Axial sections of CVR maps (upper row) with the color scale are shown on the right (percentage change of BOLD per millimeter-of-mercury change in PetCO $\mathrm{C}_{2}$ ). $A, \mathrm{CVR}$ map 1 month prior to surgery; $B, C V R$ measured 4 months postoperatively; $C$, second follow-up CVR measured 8 months postoperatively; $D$ and $E$, additional follow-up CVR maps measured 18 and 35 months postoperatively. Their corresponding CVR ID z-maps are found in the second and third rows, as indicated by the brackets. $F$ is an ID z-map that compares study A with study E, or the net change in CVR for the entire duration. See text for interpretations.

surements were more physiologic in nature, reflecting the balance of blood flow resulting from the establishment of spontaneously developing and surgically established collateral blood flow. In these patients, the CVR ID z-maps indicated the significant changes in CVR with time, adding to the assessment of the success of surgical interventions. This ability is well-illustrated in the patient shown in Fig 4. In this patient, bypass revascularization surgery transiently improved CVR, but not to normal values. Thereafter, however, the CVR ID z-maps showed the degree and extent to which CVR progressively declined to a stable level at the final follow-up scan. The patient continuing to be symptomatic is also consistent with the CVR scans.

\section{Limitations}

Accurate measurement of CBF in advanced cerebrovascular disease is difficult. PET remains the criterion standard, and current MR imaging vendor implementations of arterial spin-labeling have yet to be proved/validated for clinical use. ${ }^{28}$ The use of BOLD as a surrogate measure of CBF is common and has been shown to correlate well with other modalities, including FAIR arterial spin-labeling ${ }^{29}$ (also see Duffin et $\mathrm{al}^{9}$ and Tancredi et $\mathrm{al}^{30}$ for discussions). Nevertheless, the relationship between blood flow and BOLD is nonlinear. ${ }^{31,32}$ The atlas approach helps to mitigate this issue. In this article, the $z$ scores account for the limitations of BOLD reflecting CBF because this measure of CBF was used in both the patient studies and the atlas. As improved methods of measuring CBF become available, the same analysis methodology can also be applied.

Within-subject variability of CVR may differ more with age and sex than in the men in our reference cohort. ${ }^{33}$ In this study, we examined male subjects and used only men for our reference atlas. Nevertheless, we note that the between-day differences in CVR in women studied with a methodology similar to ours found no significant interval differences. ${ }^{17}$ Our reference CVR ID atlas was not selected for a specific age group (mean age, $36.7 \pm 16.1$ ), and neither was our patient cohort. The effect of age on CVR is unclear. Some studies found reductions in $\mathrm{CVR}^{34,35}$; we and others found no correlation between age and CVR. ${ }^{27,36}$ However, the question is not whether age is a factor in CVR but whether age is a factor in test-retest differences obtained within a matter of a few months. There is no reason to suppose that interval differences in healthy subjects differ greatly with age.

Another limitation to the sensitivity of this study is the variation in the time differences between measurements. Test-to-test differences due to the variable duration between tests are probably of the same order of magnitude as those due to discrepancies in sex, comorbidities, or other anthropomorphic factors. Such unaccounted for discrepancies will further reduce the sensitivity and increase specificity of the atlas by reducing the ID $\mathrm{z}$-values.

In our CVR ID z-map approach, it is assumed that the range of test-to-test variability in CVR found in the abnormal areas of our patient group is the same as that in healthy tissue. We therefore assume that any changes in the abnormal tissue CVR with time due to a pathological process will be greater than changes in normal tissue, ensuring the ability of the CVR ID z-maps to distinguish true pathologic changes in abnormal areas. Nevertheless, further investigation may be required to determine the "normal" variability in abnormal areas. For this measurement, patient scans should be repeated within a short time (eg, $<1$ week), within which no substantial change would be expected in, for example, patients who are stable and not candidates for revascularization. Their CVR ID z-maps would reflect the normal variability in abnormal areas. Such measurements are shown in Fig 4, where CVR ID z-maps show no changes between $C$ and $D$, and $D$ and $E$.

Finally, a key confounding factor that affects CVR interpretation is blood pressure. ${ }^{37}$ However, we were not able to measure blood pressure due to the constraints imposed by the MR imaging environment on this measurement. Accurate frequent blood pressure sampling in the MR imaging environment would aid in assessing the sensitivity of the CVR ID $z$ scoring in the future.

\section{CONCLUSIONS}

CVR ID z-maps are a secondary analysis of CVR maps that provides a statistical approach to separate test-to-test differences due to technical and physiologic changes with time from those due to changes in the underlying pathophysiology, informing the clinical assessment of disease activity and the response to treatment. The patients we present underwent considerable 
changes in CVR. The lower the likelihood that these changes represent test-to-test variability, the more likely they are to represent underlying pathophysiology, despite few overt changes in symptoms. We therefore suggest that the advanced analysis of the CVR data by using CVR ID z-maps introduced in this article can improve assessment of longitudinal subclinical pathophysiologic changes that are difficult to gauge by using angiography and CVR maps alone.

\section{ACKNOWLEDGMENTS}

The authors thank Toronto Western Hospital MR imaging technologist Eugen Hlasny for his help in acquiring the MR imaging data and clinical coordinator Abby Skanda for her help with study organization.

Disclosures: Olivia Sobczyk—UNRELATED: Consultancy/Employment: I am a consultant (part-time) of Thornhill Research, whose parent company owns intellectual property on the RespirAct device used in the study. James Duffin-UNRELATED: Employment, Patents (planned, pending or issued), Stock/Stock Options: Thornhill Research. Joseph A. Fisher-RELATED: Consulting Fee or Honorarium: The device that was used to control blood gases in the CVR experiments in this study is a custom prototype built by Thornhill Research, which is a spin-off of the University Health Network. I am also one of the inventors of this device and the chief scientist at Thornhill Research.

\section{REFERENCES}

1. Markus H, Cullinane M. Severely impaired cerebrovascular reactivity predicts stroke and TIA risk in patients with carotid artery stenosis and occlusion. Brain 2001;124:457-67 CrossRef Medline

2. Ogasawara K, Ogawa A, Yoshimoto T. Cerebrovascular reactivity to acetazolamide and outcome in patients with symptomatic internal carotid or middle cerebral artery occlusion: a xenon-133 singlephoton emission computed tomography study. Stroke 2002;33: 1857-62 CrossRef Medline

3. Sasoh M, Ogasawara K, Kuroda K, et al. Effects of EC-IC bypass surgery on cognitive impairment in patients with hemodynamic cerebral ischemia. Surg Neurol 2003;59:455-60; discussion 460-63 CrossRef Medline

4. Silvestrini M, Viticchi G, Falsetti L, et al. The role of carotid atherosclerosis in Alzheimer's disease progression. J Alzheimers Dis 2011; 25:719-26 CrossRef Medline

5. Han JS, Mandell DM, Poublanc J, et al. BOLD-MRI cerebrovascular reactivity findings in cocaine-induced cerebral vasculitis. Nat Clin Pract Neurol 2008;4:628-32 CrossRef Medline

6. Fierstra J, Poublanc J, Han JS, et al. Steal physiology is spatially associated with cortical thinning. J Neurol Neurosurg Psychiatry 2010; 81:290-93 CrossRef Medline

7. Mandell DM, Han JS, Poublanc J, et al. Quantitative measurement of cerebrovascular reactivity by blood oxygen level-dependent MR imaging in patients with intracranial stenosis: preoperative cerebrovascular reactivity predicts the effect of extracranial-intracranial bypass surgery. AJNR Am J Neuroradiol 2011;32:721-27 CrossRef Medline

8. Sobczyk O, Battisti-Charbonney A, Fierstra J, et al. A conceptual model for $\mathrm{CO}_{2}$-induced redistribution of cerebral blood flow with experimental confirmation using BOLD MRI. Neuroimage 2014;92: 56-68 CrossRef Medline

9. Duffin J, Sobczyk O, Crawley AP, et al. The dynamics of cerebrovascular reactivity shown with transfer function analysis. Neuroimage 2015;114:207-16 CrossRef Medline

10. Poublanc J, Crawley AP, Sobczyk O, et al. Measuring cerebrovascular reactivity: the dynamic response to a step hypercapnic stimulus. J Cereb Blood Flow Metab 2015;35:1746-56 CrossRef Medline

11. Sommer LZ, Iscoe S, Robicsek A, et al. A simple breathing circuit minimizing changes in alveolar ventilation during hyperpnoea. Eur Respir J 1998;12:698-701 CrossRef Medline
12. Slessarev M, Han J, Mardimae A, et al. Prospective targeting and control of end-tidal CO2 and $\mathbf{O} 2$ concentrations. J Physiol 2007;581: 1207-19 CrossRef Medline

13. Cox RW. AFNI: software for analysis and visualization of functional magnetic resonance neuroimages. Comput Biomed Res 1996; 29:162-73 CrossRef Medline

14. Saad ZS, Glen DR, Chen G, et al. A new method for improving functional-to-structural MRI alignment using local Pearson correlation. Neuroimage 2009;44:839-48 CrossRef Medline

15. Ashburner J, Friston K. Multimodal image coregistration and partitioning: a unified framework. Neuroimage 1997;6:209-17 CrossRef Medline

16. Ashburner J, Friston KJ. Nonlinear spatial normalization using basis functions. Hum Brain Mapp 1999;7:254-66 Medline

17. Kassner A, Winter JD, Poublanc J, et al. Blood-oxygen level dependent MRI measures of cerebrovascular reactivity using a controlled respiratory challenge: reproducibility and gender differences. J Magn Reson Imaging 2010;31:298-304 CrossRef Medline

18. Mark CI, Slessarev M, Ito S, et al. Precise control of end-tidal carbon dioxide and oxygen improves BOLD and ASL cerebrovascular reactivity measures. Magn Reson Med 2010;64:749-56 CrossRef Medline

19. Fierstra J, Sobczyk O, Battisti-Charbonney A, et al. Measuring cerebrovascular reactivity: what stimulus to use? J Physiol 2013;591: 5809-21 CrossRef Medline

20. Tancredi FB, Hoge RD. Comparison of cerebral vascular reactivity measures obtained using breath-holding and $\mathrm{CO} 2$ inhalation. J Cereb Blood Flow Metab 2013;33:1066-74 CrossRef Medline

21. Kety SS, Schmidt CF. The effects of altered arterial tension of carbon dioxide and oxygen on cerebral blood flow and cerebral oxygen consumption of normal young men. J Clin Invest 1948;27:484-92 CrossRef Medline

22. Markus HS, Harrison MJ. Estimation of cerebrovascular reactivity using transcranial Doppler, including the use of breath-holding as the vasodilatory stimulus. Stroke 1992;23:668-73 CrossRef Medline

23. van der Zande FH, Hofman PA, Backes WH. Mapping hypercapniainduced cerebrovascular reactivity using BOLD MRI. Neuroradiology 2005;47:114-20 CrossRef Medline

24. Silvestrini M, Vernieri F, Troisi E, et al. Cerebrovascular reactivity in carotid artery occlusion: possible implications for surgical management of selected groups of patients. Acta Neurol Scand 1999;99: 187-91 CrossRef Medline

25. Prisman E, Slessarev M, Azami T, et al. Modified oxygen mask to induce target levels of hyperoxia and hypercarbia during radiotherapy: a more effective alternative to carbogen. Int J Radiat Biol 2007;83:457-62 CrossRef Medline

26. Ito S, Mardimae A, Han J, et al. Non-invasive prospective targeting of arterial $\mathbf{P}(\mathbf{C O} 2)$ in subjects at rest. J Physiol 2008;586:3675-82 CrossRef Medline

27. Sobczyk O, Battisti-Charbonney A, Poublanc J, et al. Assessing cerebrovascular reactivity abnormality by comparison to a reference atlas. J Cereb Blood Flow Metab 2015;35:213-20 CrossRef Medline

28. Grade M, Hernandez Tamames JA, Pizzini FB, et al. A neuroradiologist's guide to arterial spin labeling MRI in clinical practice. Neuroradiology 2015;57:1181-202 CrossRef Medline

29. Mandell DM, Han JS, Poublanc J, et al. Mapping cerebrovascular reactivity using blood oxygen level-dependent MRI in patients with arterial steno-occlusive disease: comparison with arterial spin labeling MRI. Stroke 2008;39:2021-28 CrossRef Medline

30. Tancredi FB, Lajoie I, Hoge RD. Test-retest reliability of cerebral blood flow and blood oxygenation level-dependent responses to hypercapnia and hyperoxia using dual-echo pseudo-continuous arterial spin labeling and step changes in the fractional composition of inspired gases. J Magn Reson Imaging 2015;42:1144-57 CrossRef Medline

31. Davis TL, Kwong KK, Weisskoff RM, et al. Calibrated functional MRI: mapping the dynamics of oxidative metabolism. Proc Natl Acad Sci U S A 1998;95:1834-39 CrossRef Medline

32. Hoge RD, Atkinson J, Gill B, et al. Investigation of BOLD signal 
dependence on cerebral blood flow and oxygen consumption: the deoxyhemoglobin dilution model. Magn ResonMed 1999;42:849-63 Medline

33. Kastrup A, Thomas C, Hartmann C, et al. Sex dependency of cerebrovascular CO2 reactivity in normal subjects. Stroke 1997;28: 2353-56 CrossRef Medline

34. Flück D, Beaudin AE, Steinback CD, et al. Effects of aging on the association between cerebrovascular responses to visual stimulation, hypercapnia and arterial stiffness. Front Physiol 2014;5:49 CrossRef Medline
35. Mark CI, Mazerolle EL, Chen JJ. Metabolic and vascular origins of the BOLD effect: implications for imaging pathology and restingstate brain function. J Magn Reson Imaging 2015;42:231-46 CrossRef Medline

36. Schwertfeger N, Neu P, Schlattmann P, et al. Cerebrovascular reactivity over time course in healthy subjects. J Neurol Sci 2006;249: 135-39 CrossRef Medline

37. Regan RE, Fisher JA, Duffin J. Factors affecting the determination of cerebrovascular reactivity. Brain Behav 2014;4:775-88 CrossRef Medline 